\title{
Facile method to align crystalline needles composed of organic semiconducting materials using a balance between centrifugal and capillary forces
}

\author{
MASASHI WATANABE* ${ }^{\circ}$ and KOUHEI TANAKA \\ Faculty of Textile Science and Technology, Shinshu University, 3-15-1 Tokida, Ueda, Nagano 386-8567, Japan \\ *Author for correspondence (mwatana@shinshu-u.ac.jp)
}

MS received 14 December 2016; accepted 27 February 2017; published online 23 September 2017

\begin{abstract}
Methods to align one-dimensional microstructures composed of organic materials have attracted much attention because of their potential applications to devices such as field-effect transistors. Although dip coating is one of the methods (using self-assembly) used for the purpose, its disadvantage is that a larger amount of the material dissolves in the solution than what actually gets deposited on substrate. In this study, we developed a novel method that requires a small amount of precursor solution. By placing a polydimethylsiloxane block on a glass substrate and rotating the substrate using a spin coater, a small amount of the precursor solution was confined in a narrow region along the foot of the block. When we used an organic semiconducting material, 9,10-dibromoanthracene, as a solute, aligned and roughly oriented crystalline needles were precipitated. The thicknesses and lengths of the crystalline needles were controlled by the composition of the solvent and the rotation speed.
\end{abstract}

Keywords. Crystalline needles; centrifugal force; capillary force; organic semiconducting materials; self-assembly.

\section{Introduction}

Aligned one-dimensional micrometre- or nanometre-sized structures have recently attracted much attention [1] because of the diversity of their potential applications, such as fieldeffect transistors [2], light-emitting diodes [3], solar cells [4] and biochemical sensors [5]. In the case where a large number of nanowires need to be aligned, methods based on selfassembly seem to be more advantageous than manipulating them one by one [6]. Dip coating is one such method [7,8]. However, the amount of nanowires deposited (or precipitated) on a substrate is much smaller than that of nanowires initially dispersed in the suspension (or that of materials initially dissolved in the solution). Thus, a majority of the nanowires is left in the suspension. This is not desirable from the points of view of cost-effectiveness, resource conservation and waste reduction. Especially in laboratory scale experiments, this issue may have a larger impact because each suspension is usually used only for a few times.

To address this problem by using as little solution (or suspension) as possible, the solution should be confined to a small area on the substrate. A hydrophilic area surrounded by a hydrophobic one can confine an aqueous solution, which can be used to produce organic semiconducting single crystals [9]. However, it requires patterning the wettability of the surface using microcontact printing or conventional photolithography. A small, protruded object, such as a convex lens, placed on a flat substrate also confines fluid under it. This method can be used to form concentric thin lines made from a polymer [10]. In this method, a small amount of solution (e.g., $25 \mu \mathrm{l}$ ) should be carefully loaded into a submillimetre space between the object and the substrate before letting them come into contact with each other. Another method uses microchannels composed of a glass substrate and polydimethylsiloxane (PDMS) mould. The channels can confine precursor solutions, and, thus, the method can be used for forming nanowire patterns [11]. However, evaporation of the solvent is possible only through the open end of the channel, suggesting that a vacuum condition or a long time is required to dry the solution.

In this study, we propose the following method using a PDMS block placed on the substrate in order to confine a solution: a small amount of the solution is poured on the side of the block (figure 1a). Using centrifugal force, excess solution is removed and some portion of the solution remains beside on the block due to capillary force (figure 1b). The amount of the remaining solution is determined by a balance between centrifugal force and capillary force. Finally, crystalline needles composed of the solute are formed as the solution dries (figure 1c). In the study, we used 9,10-dibromoanthracene (DBA), which is an organic semiconducting material [12], as the solute to form crystalline needles of DBA and orient them along a preferred direction.

Li et al [13] reported the use of a small piece of silicon wafer $\left(0.4 \times 0.4 \mathrm{~cm}^{2}\right)$, instead of the PDMS block, for single-crystalline needle alignment. In their method, a 
(a)

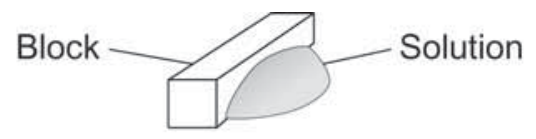

(b)

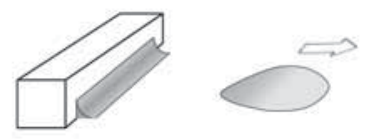

(c)

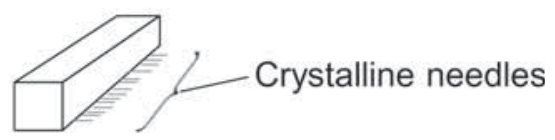

Figure 1. Working hypothesis for aligning crystalline needles using a small amount of solution. (a) A small amount of solution is first dropped near a block. (b) Excess solution is removed by centrifugal force. (c) As the solution dries, crystalline needles will form along the block.

solution droplet $(20 \mu \mathrm{l})$ was first placed on the substrate, and then a piece of silicon wafer was placed over the droplet. Consequently, the solution that was forced out from beneath the wafer dried and precipitated as crystalline needles. However, in their method, the control of the shape and size of the portion of the solution that is forced out is difficult. In contrast, the use of the process proposed by us in the current paper provides a way to confine the solution in a well-controlled area.

This method is expected to form micro- or nanowires at a desired position on the substrate by placing the block on that position. The lengths and orientations of the wires are also anticipated to be controlled because the wires are formed from the solution that is confined to a narrow region beside the block. Thus, the method proposed in this paper requires a small amount of solution; i.e., it is cost-effective. Therefore, the method is expected to be used to fabricate various devices such as field-effect transistors and biosensors.

\section{Experiment}

\subsection{Preparation of the PDMS block}

The precursor of PDMS (KE-103) and the curing agent (CAT-103) were purchased from Shin-Etsu Chemical (Tokyo, Japan). The precursor and the curing agent were mixed at a mass ratio of 40:1 and degassed in vacuum. This mixture was poured into a silicone rubber frame placed on a glass slide that was pre-treated with 1,1,1,3,3,3-hexamethyldisilazane (HMDS), as shown in figure 2. The mixture was then vulcanized at room temperature for 3 days. The obtained PDMS sheet was $2 \mathrm{~mm}$ thick and slightly sticky. PDMS blocks $2 \mathrm{~mm}$ wide, $15 \mathrm{~mm}$ long and $2 \mathrm{~mm}$ high were cut from this sheet. The side surface of the block was slightly (a few degrees) tilted (see the illustration in the circle of figure 2), so that the foot of the block could be easily observed from the top using a light microscope.

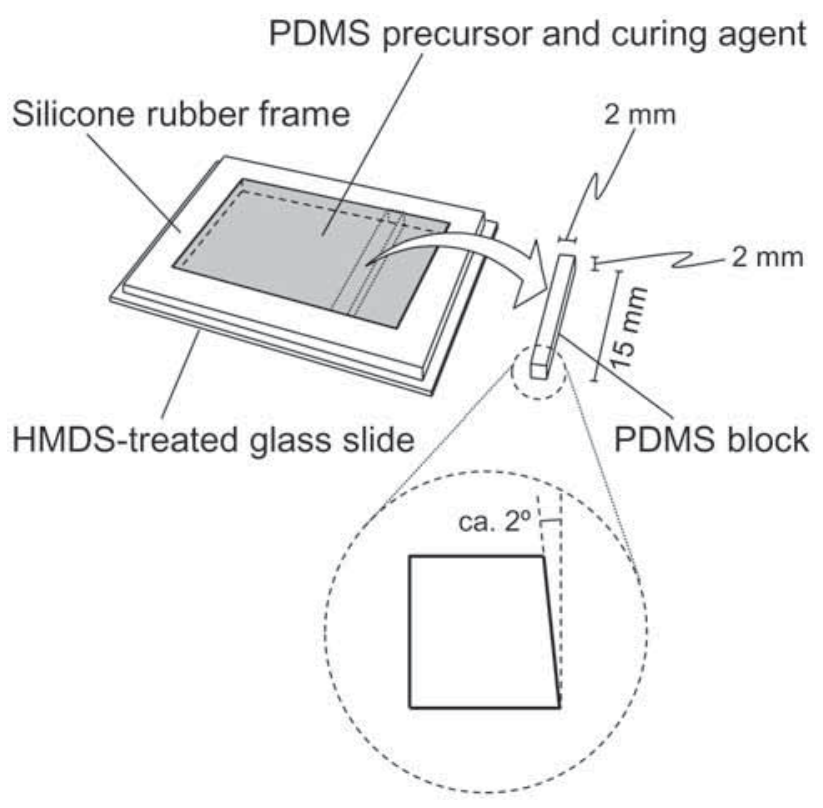

Figure 2. Preparation method of a PDMS sheet and a PDMS block cut out of the sheet.

\subsection{HMDS treatment of glass slides}

Glass slides $13 \mathrm{~mm}$ wide, $38 \mathrm{~mm}$ long and $1 \mathrm{~mm}$ thick (S-1215, Matsunami Glass Ind., Ltd., Osaka, Japan) were immersed in HMDS at room temperature for 3 days. The slides were rinsed with acetone and dried at $100^{\circ} \mathrm{C}$ for $1 \mathrm{~h}$.

\subsection{Preparation of solution of organic compound}

To obtain crystalline needles by drying solution, DBA (Wako Pure Chemical Industries, Ltd., Osaka, Japan) was dissolved in tetrahydrofuran (THF) or a mixture of THF and methanol. Because DBA seemed to be somewhat unstable in THF, the solution was used within $1 \mathrm{~h}$ of preparation. When we stored the solution for a few days at room temperature and then dried it, the morphology of the precipitated crystals was different from that obtained from freshly prepared solution. Anthraquinone (Wako Pure Chemical Industries, Ltd.) and perylenetetracarboxyldiimide (PTCDI, Sigma-Aldrich, St. Louis, MO, USA) were also employed as solutes.

\subsection{Formation of crystalline needles}

An HMDS-treated glass slide, on which a PDMS block was obliquely placed, was fixed on the rotational table of a spin coater (Mikasa Co., Ltd., Tokyo, Japan) using a double-sided tape, as shown in figure 3a. A small amount of solution $(\sim 50 \mu \mathrm{l})$, such as the solution of DBA dissolved in THF, was dropped in front of the block (figure 3b). Subsequently, the sample was rotated at a constant speed to precipitate crystalline needles of the solute (figure 3c). 
(a)

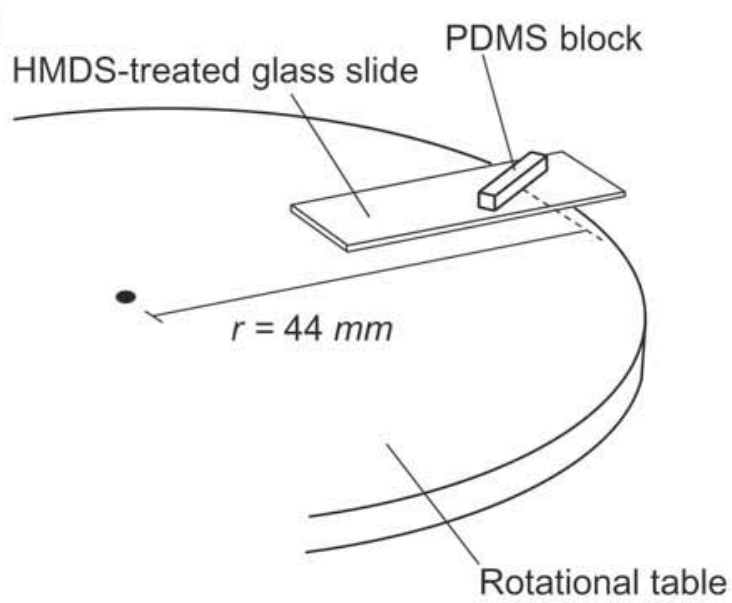

(b)

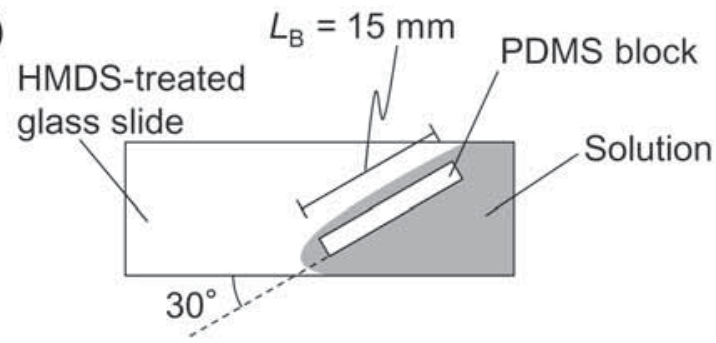

(c)

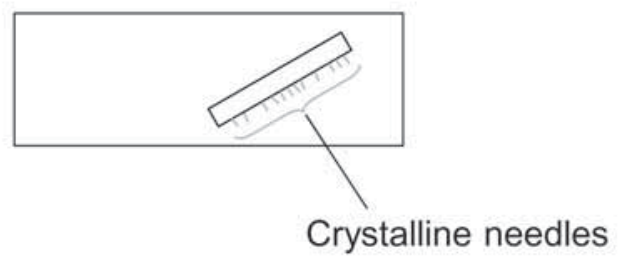

(d)

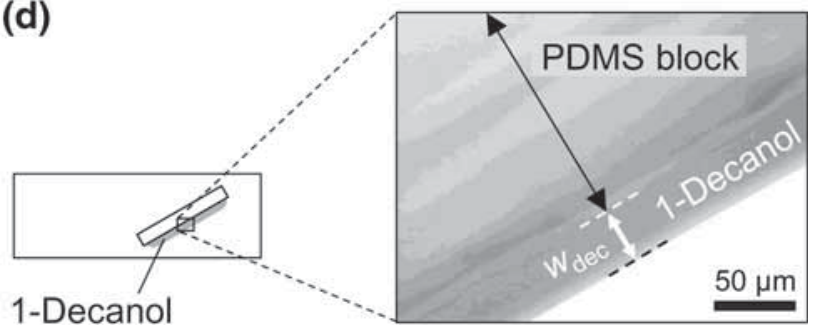

Figure 3. (a-c) Schematic diagram showing the method used to prepare aligned crystalline needles. (a) An HMDS-treated glass slide with a PDMS block is fixed on the rotational table of a spin coater. (b) A small amount of solution is then dropped at the side of the PDMS block, ensuring a contact along the side-edge. (c) When the table is rotated, crystalline needles form on the edge of the block. (d) Experimental set-up used for neat 1-decanol (left). Some amount of 1-decanol was first placed on the glass slide; excess amount of 1-decanol was then removed by rotating the glass slide. A light microscope image (right) shows the remaining 1-decanol.

\subsection{Light microscope observation of crystalline needles}

The crystalline needles were observed using the light microscope (MELUX-2-1L, Kyowa Optical Co., Ltd., Kanagawa,
Japan) with a USB image capture device (VCE-1, $300 \mathrm{kpx}$, Shodensha, Inc., Osaka, Japan). Polarizing optical microscope images were also collected using a BX51 N-33P-OC microscope (Olympus Corporation, Tokyo, Japan).

\subsection{Experiment using nonvolatile liquid}

To clarify how the length of the crystalline needles was controlled, 1-decanol was used as a nonvolatile organic liquid, instead of solution composed of low boiling-point solvents, such as THF and methanol. The experimental procedure was similar to that illustrated in figure $3 \mathrm{a}, \mathrm{b}$ and c. First, 1-decanol was dropped on the HMDS-treated glass slide. By rotating the glass slide, most of the 1-decanol was spun off; a small amount of 1-decanol remained in a belt-like region along the side-edge of the PDMS block (figure $3 \mathrm{~d}$ ). The width $w_{\mathrm{dec}}$ of this region was obtained from the light microscope images.

\subsection{Measurements of contact angles}

The advancing and receding contact angles were measured using a home-made equipment. A drop of the sample $(\sim 10 \mu \mathrm{l})$ was placed on a substrate (HMDS-treated glass slide or PDMS sheet). The substrate was then gradually tilted until the droplet began to slide down. The advancing and receding contact angles were obtained from the side-view images of the droplet captured using a CCD camera (L-800, Hozan Tool Industrial Co., Ltd., Osaka, Japan).

Static contact angles were measured by placing a droplet ( $\sim 3 \mu$ l) of the sample (e.g., THF) on a substrate. Because THF is volatile, the side-view of the droplet was filmed using a CCD camera and a video capture, instead of being photographed. A frame that was an image of the droplet before evaporation was extracted from the video and used to measure the contact angle.

\subsection{Measurements of the X-ray diffraction patterns}

$\mathrm{X}$-ray diffraction (XRD) measurements were performed using a benchtop X-ray diffractometer (MiniFlex 300, Rigaku Corporation, Tokyo, Japan) with $\mathrm{Cu} \mathrm{K} \alpha$ radiation.

\section{Results and discussion}

\subsection{Formation of crystalline needles by drying solution on the rotating glass slide}

A saturated solution of DBA dissolved in THF-methanol (1:1 in mass ratio) was dropped on the HMDS-treated glass slide. By rotating the glass slide using a spin coater, an excess amount of the solution was spun off and the remaining solution was simultaneously dried. The precipitated crystalline needles were aligned along the side- edge of the PDMS block, and they were oriented roughly perpendicular to the block (figure 4). Saturated solution of anthraquinone dissolved in 

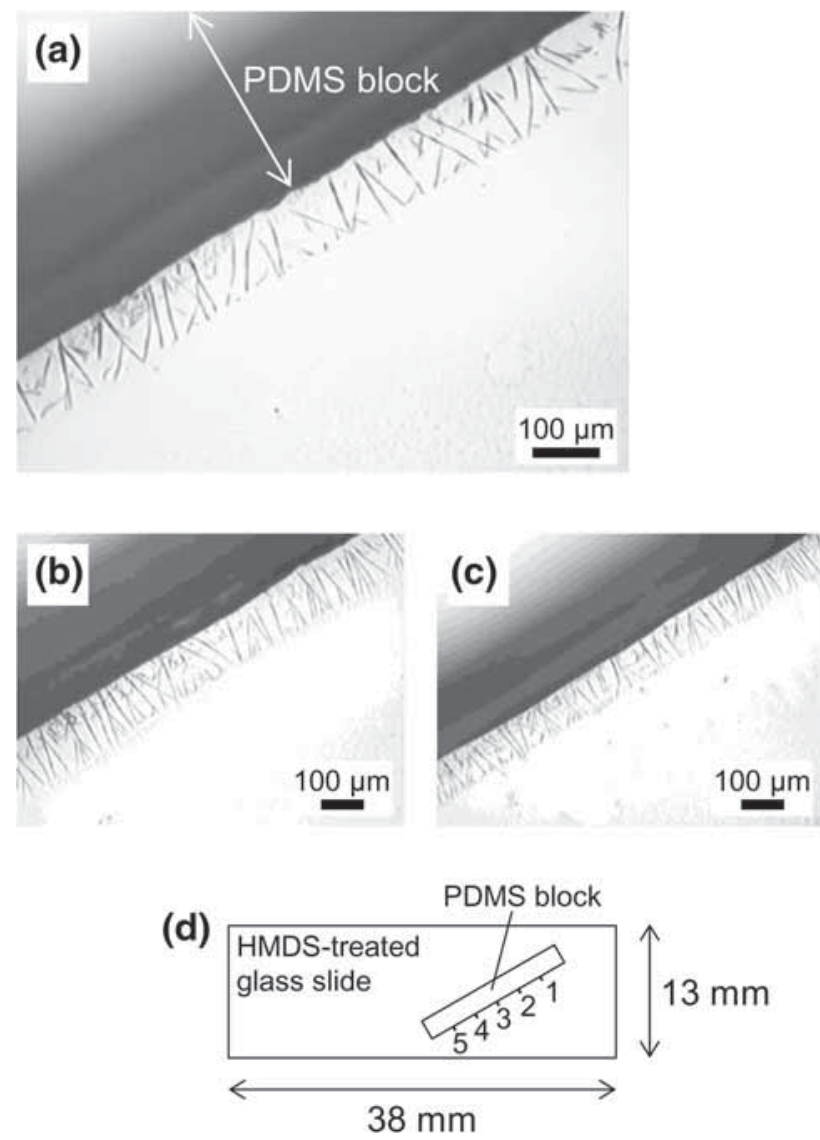

Figure 4. (a-c) Light microscope images of the aligned crystalline needles of DBA. These needles were precipitated by drying saturated solution using a spin coater. The solvent was THF-methanol (1:1 in mass ratio). The rotation speed and time were $350 \mathrm{rpm}$ and $20 \mathrm{~s}$, respectively. (d) Five regions on the glass slide observed using a light microscope. Images (a), (b) and (c) correspond to the regions numbered as 3,1 and 5, respectively.

THF-acetonitrile (17:3 in mass ratio) gave similar results (figure 5a). When $0.027 \mathrm{wt} \%$ PCTDI in chloroform-methanol (5:1 in mass ratio), which was recently noticed as a useful semiconducting organic material, was used, short crystalline needles aligned along a few lines (figure $5 \mathrm{~b}$ ). These lines seemed to be traces arising from stick-slip motion of the contact line. However, most of the precipitate seemed to be amorphous (figure 5c).

Effects of solvent on the morphology of the precipitated DBA crystals were examined. The mass ratios of THF to methanol used in this experiment were 10:0, 7:3, 5:5 and 3:7. DBA is soluble in THF up to $\sim 2 \mathrm{wt} \%$, but insoluble in methanol. Therefore, an increase in methanol content means a decrease in DBA concentration in the saturated solution. As shown in figure 6, the higher the THF content, the thicker the precipitated needle. The thinnest needle was $\sim 2 \mu \mathrm{m}$ in width, which was obtained using saturated DBA solution with a THF-methanol ratio of 5:5. Thus, the thickness of the precipitated crystalline needle could be controlled by varying
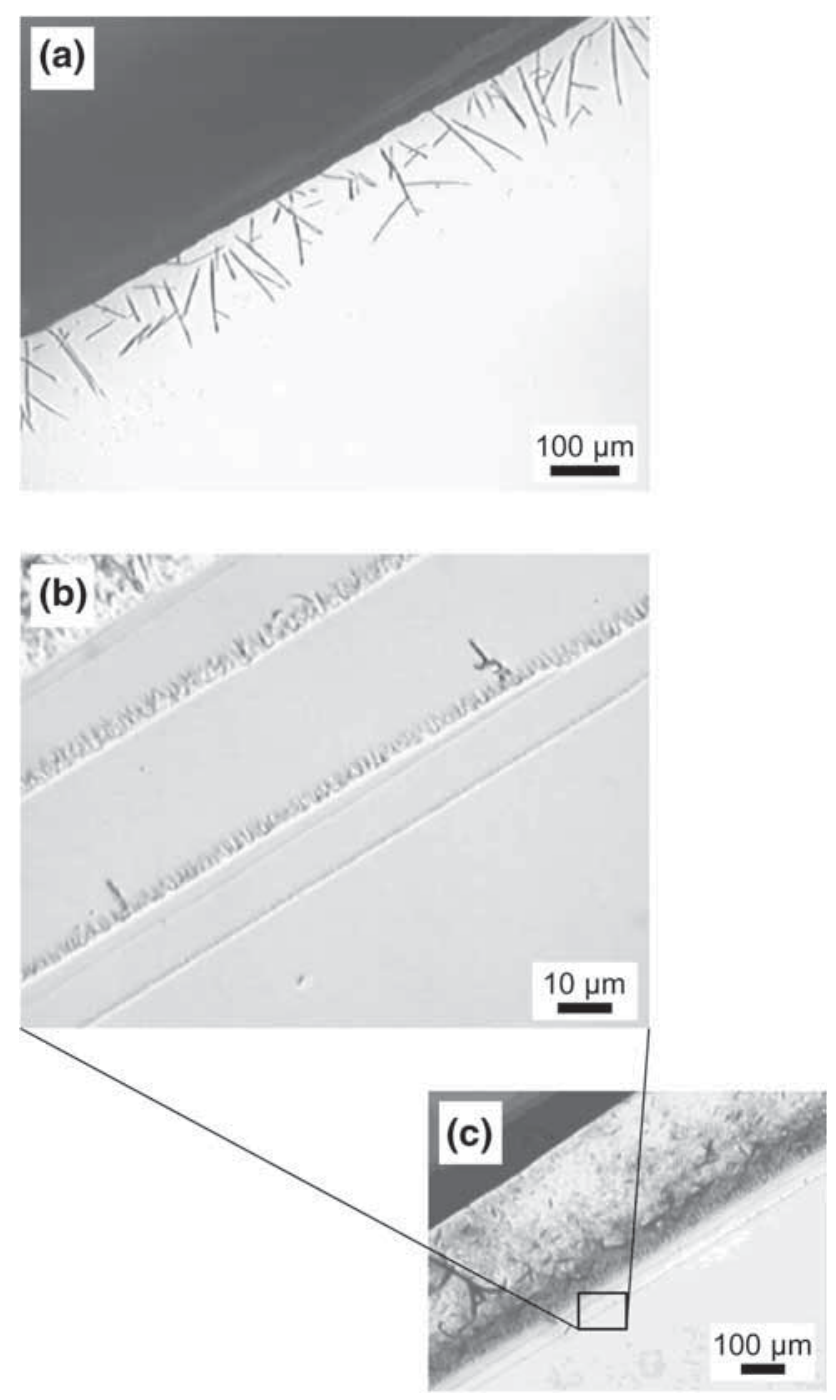

Figure 5. Light microscope images of crystalline needles generated from (a) solution (THF:acetonitrile $=17: 3$ in mass ratio) of anthraquinone and $(\mathbf{b}, \mathbf{c})$ solution (chloroform:methanol $=5: 1$ in mass ratio) of perylenetetracarboxyldiimide. Rotation speed and time were, respectively, $700 \mathrm{rpm}$ and $20 \mathrm{~s}$ for (a), and $80 \mathrm{rpm}$ and $60 \mathrm{~s}$ for (b) and (c).

the composition of mixed solvent. In addition, crossed Nicols images obtained using a polarizing optical microscope indicated that the precipitated needles were highly crystalline (figure 7). The XRD measurements also suggested that the precipitated DBA was crystalline in nature (figure 8).

We also tried to control the length of the crystalline needles by changing rotation speed. The solvent used to dissolve DBA was THF-methanol (1:1 in mass ratio). As shown in figure 9, a lower speed tended to give longer crystalline needles. The distance between the outer tip of the crystalline needle (i.e., the tip farther from the rotation axis) and the PDMS block, $L_{\mathrm{DBA}}$, was plotted against rotation speed (figure 10a). According to the $\log -\log$ chart (figure $10 \mathrm{~b}$ ), $L_{\mathrm{DBA}}$ is proportional to $\omega^{-0.52}$, where $\omega$ is the angular frequency. 

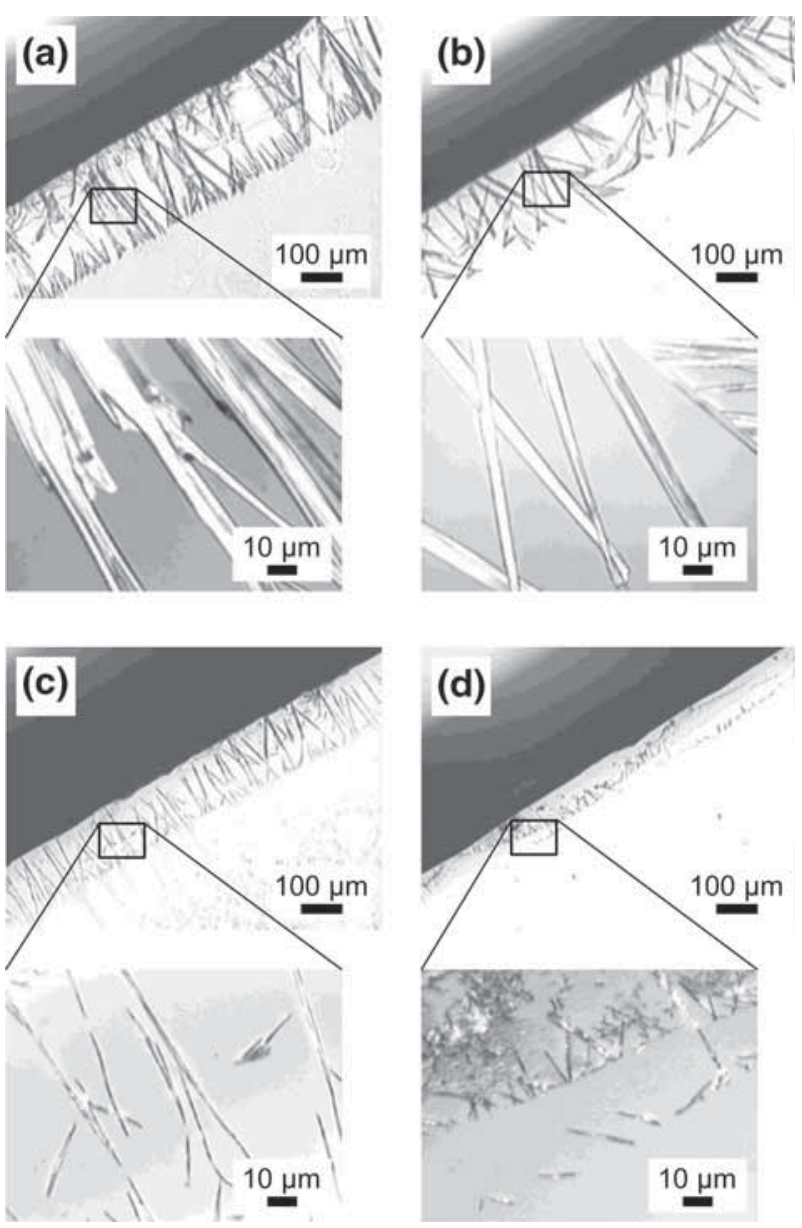

Figure 6. Light microscope images of crystalline DBA needles. The thickness of these needles was controlled by changing the composition of the solvent. The mass ratios of THF to methanol were (a) 10:0, (b) 7:3, (c) 5:5 and (d) 3:7. Rotation speed and time were $200 \mathrm{rpm}$ and $20 \mathrm{~s}$, respectively.

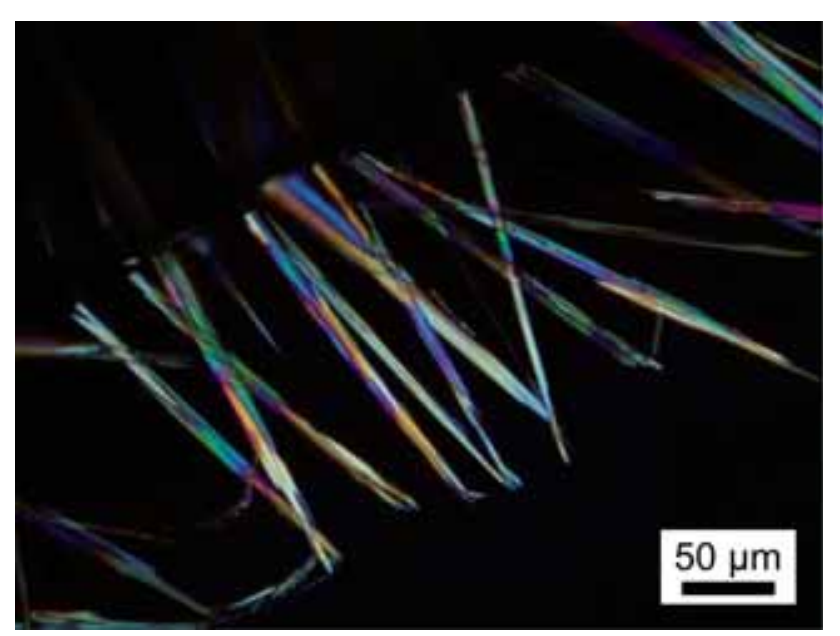

Figure 7. Crossed Nicols image of crystalline needles precipitated from DBA solution. The mass ratio of the solvents THF-methanol was 7:3. Rotation speed and time were $200 \mathrm{rpm}$ and $20 \mathrm{~s}$, respectively.

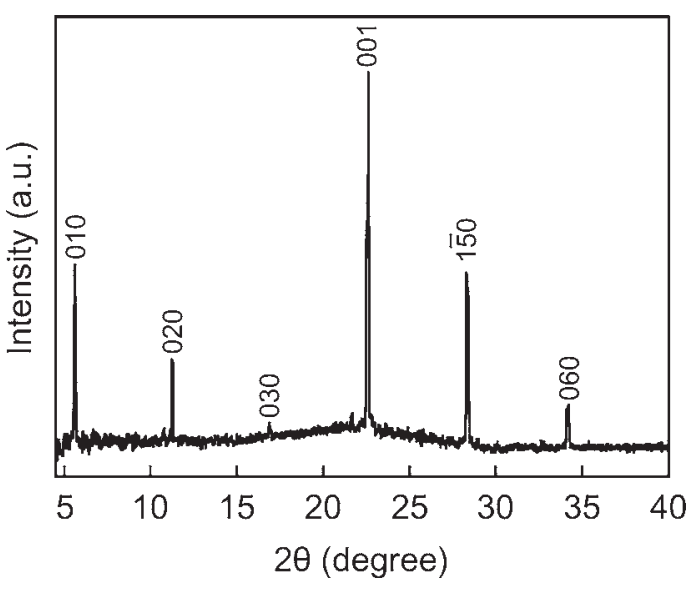

Figure 8. XRD pattern of crystalline DBA needles.

\subsection{Mechanism of the orientation of crystalline needles}

As reported by Deegan et al [14], while a droplet of aqueous solution dries, the solution flows outwards from the centre of the droplet. Huang et al [7] employed such a flow to align nanowires contained in dispersion. During the drying of an octane droplet, Marangoni convection was found to occur because of the latent heat of evaporation, as reported by $\mathrm{Hu}$ and Larson [15]. In addition, the evaporation flux was found to be higher at the edge of the droplet [16].

We prepared a saturated solution of anthraquinone using acetonitrile as the solvent. A droplet of the solution was placed on the HMDS-treated glass slide and observed using the light microscope. During the observation, the glass slide was stored in a closed Petri dish to remove the effect of the outside air current. As the droplet dried, crystalline needles were formed mainly at the edge of the droplet and roughly oriented to the direction perpendicular to the contact line (figure 11a). Some crystals formed in the interior and drifted towards the droplet edge.

Based on this observation, we depict a plausible mechanism in figure $11 \mathrm{~b}$. Crystalline needles mainly begin to grow at the edge of the droplet because the evaporation flux is the highest there. Therefore, one end of the crystal is anchored to the glass slide at the droplet edge. The other end is oriented due to the Marangoni convection. Thus, crystalline needles are aligned along the contact line and oriented to the direction perpendicular to this line.

\subsection{Mechanism for regulating lengths of the crystalline needles}

Crystalline needles were plausibly formed through the following two steps: (1) excess solution was removed by the centrifugal force and a small amount of solution was left along the PDMS block and (2) the solvent was then (or simultaneously) evaporated into surrounding air to precipitate crystals. 


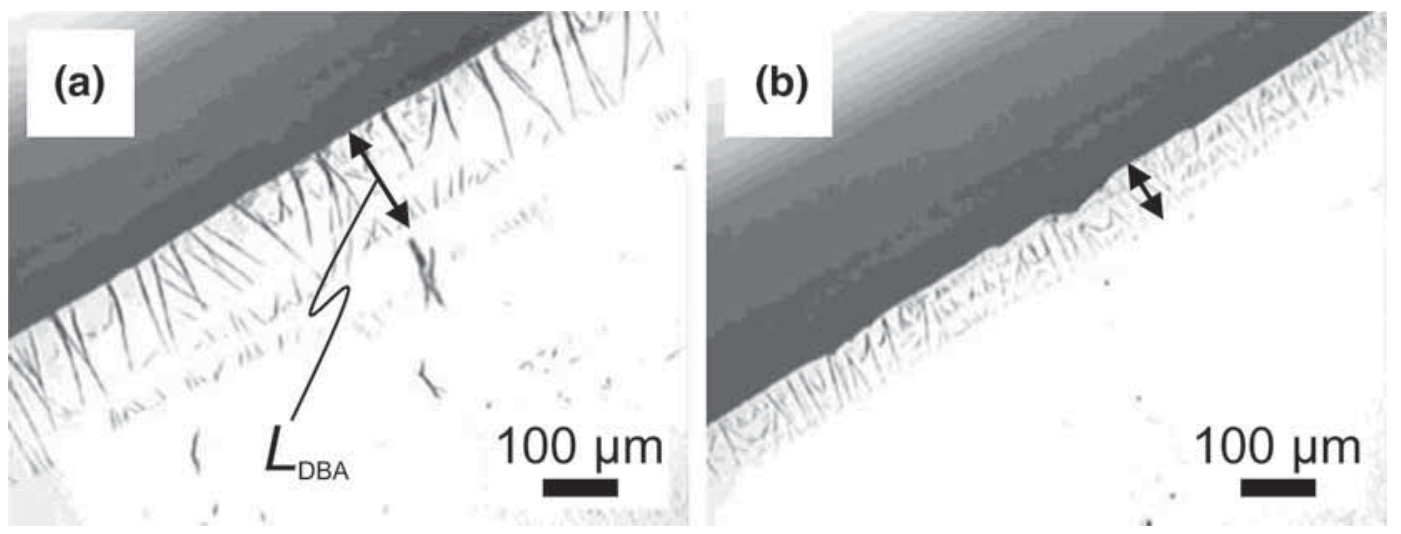

Figure 9. Light microscope images of crystalline DBA needles whose length was varied by rotation speed of the spin coater at (a) 200 and (b) $650 \mathrm{rpm}$. The solvent was THF-methanol in a 1:1 mass ratio.
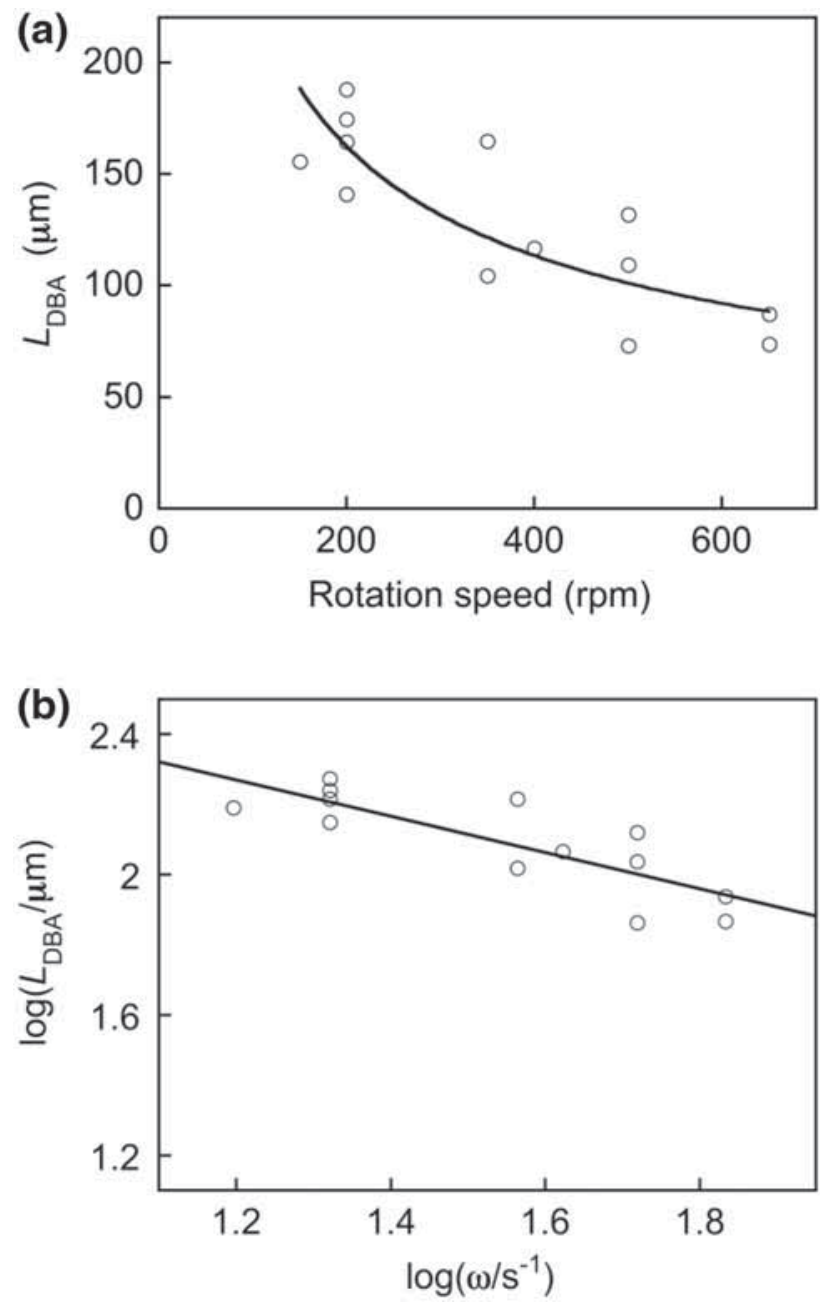

Figure 10. (a) Relationship between rotation speed and length of crystalline DBA needles. The solvent was THF-methanol (1:1 in mass ratio); (b) log-log chart based on the data shown in $\mathbf{a}$.

Watanabe et al [17] reported that some of the commonly used solvents, such as chloroform, can gradually evaporate through PDMS. However, because of the different geometry of the
PDMS block and the substrate used in this study, the major part of the solvent was directly evaporated into air.)

Here, we consider a model for the first step, as shown in figure 12. It is plausible that the centrifugal force balances capillary forces exerted through the contact line on the PDMS block or the glass slide. In the directions perpendicular and parallel to the longitudinal direction of the PDMS block, the centrifugal force balances capillary forces, as shown in figure $12 \mathrm{a}$ and $\mathrm{b}$, respectively. The balance in the perpendicular direction can be expressed by the relation

$$
L_{\mathrm{B}} \gamma \sin \theta_{2}=m r \omega^{2} \sin 30^{\circ}+L_{\mathrm{B}} \gamma \cos \theta_{1},
$$

where $L_{\mathrm{B}}$ is the length of the PDMS block, $\gamma$ is the surface tension of the solution, $m$ is the mass of the solution, $r$ is the distance from the spin axis, $\omega$ is the angular frequency and $\theta_{1}$ and $\theta_{2}$ are the contact angles. In this equation, the centrifugal force is written as $m r \omega^{2} \sin 30^{\circ}$ because the PDMS block is not parallel to the radial direction. When $m$ is its maximum value, $\theta_{1}$ is equal to the advancing contact angle $\theta_{\mathrm{A}}$, and $\theta_{2}$ is a right angle. Therefore, $m$ should satisfy the following inequality:

$$
m \leqq \frac{L_{\mathrm{B}} \gamma\left(1-\cos \theta_{\mathrm{A}}\right)}{r \omega^{2} \sin 30^{\circ}} .
$$

Similarly, the balance in the parallel direction can be written as

$$
\begin{aligned}
\left(w_{1}+w_{2}\right) \gamma \cos \theta_{4}= & m r \omega^{2} \cos 30^{\circ} \\
& +\left(w_{1}+w_{2}\right) \gamma \cos \theta_{3},
\end{aligned}
$$

where $w_{1}$ is the length of contact line on the glass slide and $w_{2}$ is that on the side of the PDMS block, as shown in figure 12a. As shown in figure $12 \mathrm{~b}, \theta_{3}$ and $\theta_{4}$ are the contact angles. This equation is similar to Furmidge's equation for a droplet slid by gravity [18]. Although the contact angle on the glass slide was 
(a)

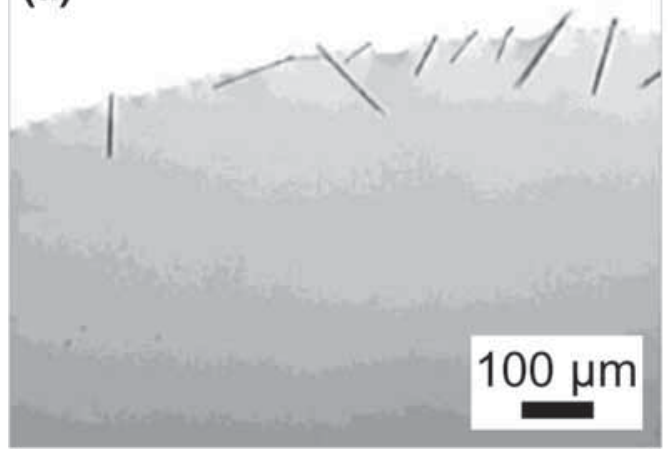

(b)

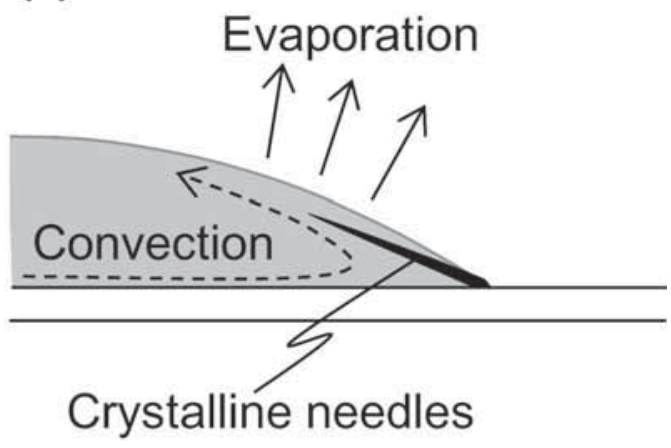

Figure 11. (a) Light microscope image of crystalline needles generated at the edge of a droplet of acetonitrile solution of anthraquinone. (b) Plausible mechanism to orient crystalline needles.

(a) Cross-section

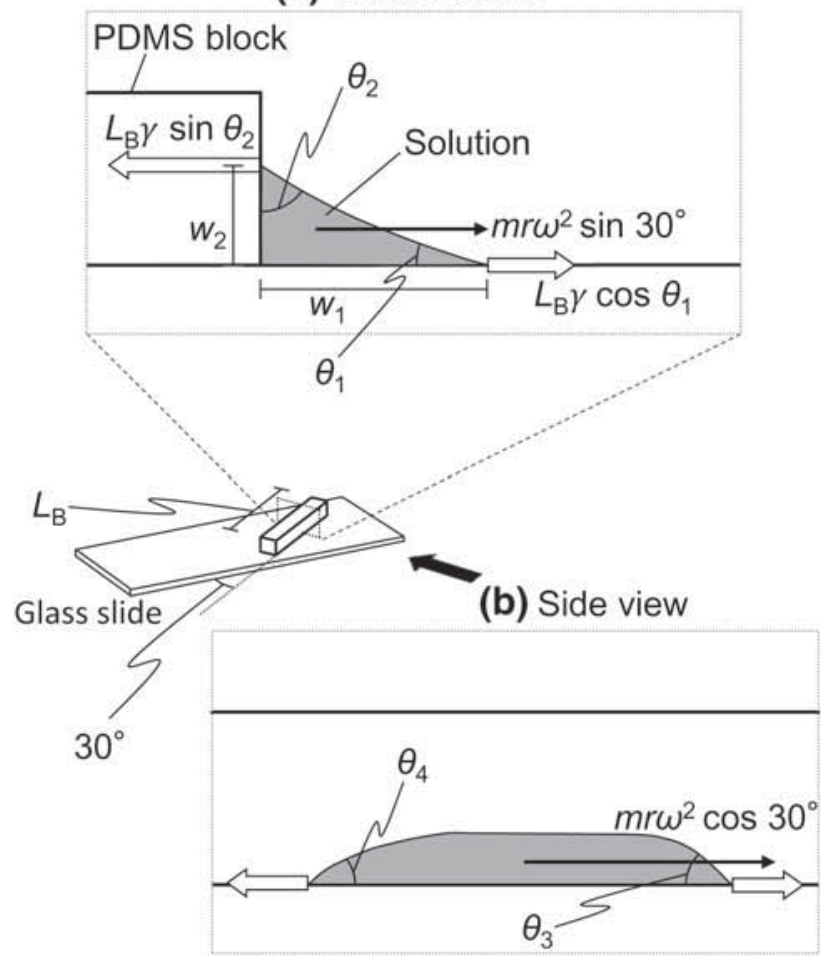

Figure 12. Schematic illustration of forces exerted on solution remaining along the foot of the PDMS block. (a) Cross-section and (b) side view of the block.

actually different from that on the PDMS block, we assumed that they were equal to each other to simplify the equation.

When the solution remaining beside the block is not displaced by centrifugal force, $\theta_{3}$ should be smaller than the advancing contact angle, $\theta_{\mathrm{A}}$, and $\theta_{4}$ should be larger than the receding contact angle, $\theta_{\mathrm{R}}$. Therefore, the following inequality can be written from equation (3):

$$
m \leqq \frac{\left(w_{1}+w_{2}\right) \gamma\left(\cos \theta_{\mathrm{R}}-\cos \theta_{\mathrm{A}}\right)}{r \omega^{2} \cos 30^{\circ}} .
$$

Here, $w_{1}$ is supposed to be a few hundred micrometres, based on the length of the obtained crystalline needles (figure 9), and $w_{2}$ must be smaller than the height of the PDMS block ( $2 \mathrm{~mm}$ ). The length of the PDMS block $L_{\mathrm{B}}$ is $15 \mathrm{~mm}$. Thus, $\left(w_{1}+w_{2}\right)$ is much smaller than $L_{\mathrm{B}}$, indicating that $m$ can be determined by equation (4) rather than equation (2). The maximum value of $m$ in equation (4) can be the amount of the solution left after excess solution was spun off, which we write here as $m_{\text {left }}$.

To roughly understand the relationship between the length $w_{1}$ and the angular frequency $\omega$, the solution is assumed to be confined in a triangular prism where $w_{1}$ is equal to $w_{2}$. Thus, the volume of the triangular prism is $w_{1}^{2} L_{\mathrm{B}} / 2$ and the amount of the solution $m_{\text {left }}$ can be written as $w_{1}^{2} L_{\mathrm{B}} \rho / 2$, where $\rho$ is the density of the solution. Therefore, from equation (4), $w_{1}$ can be rewritten as follows:

$$
w_{1}=\frac{4 \gamma\left(\cos \theta_{\mathrm{R}}-\cos \theta_{\mathrm{A}}\right)}{L_{\mathrm{B}} \rho r \omega^{2} \cos 30^{\circ}} .
$$

Thus, $w_{1}$ is proportional to $\omega^{-2}$.

This relationship was verified using 1-decanol (boiling point: $233^{\circ} \mathrm{C}$ ) as a substantially nonvolatile organic liquid instead of the solution composed of THF and methanol (boiling points: 66 and $64.7^{\circ} \mathrm{C}$, respectively). As shown in figure $3 \mathrm{~d}$, 1-decanol was poured on a glass slide and the width $w_{\text {dec }}$ of the belt-like region, where 1-decanol remained, was measured. Prior to experiments of varying the rotation speed, we examined the relationship between $w_{\mathrm{dec}}$ and rotation time using a constant rotation speed $(600 \mathrm{rpm})$. The width $w_{\text {dec }}$ was roughly constant for the rotation times ranging from 30 to $100 \mathrm{~s}$, indicating that evaporation of 1-decanol was negligible during the rotation (figure 13).

The dependence of $w_{\mathrm{dec}}$ on rotation speed was revealed under the condition of a constant rotation time ( $50 \mathrm{~s}$ ), as shown in figure 14a. By considering the slope of a log-log chart, $w_{\mathrm{dec}}$ was found to be proportional to $\omega^{-1.84}$ (figure 14b). Thus, the relationship is roughly consistent with the afore-mentioned theory, where $w_{1}$ is proportional to $\omega^{-2}$. 


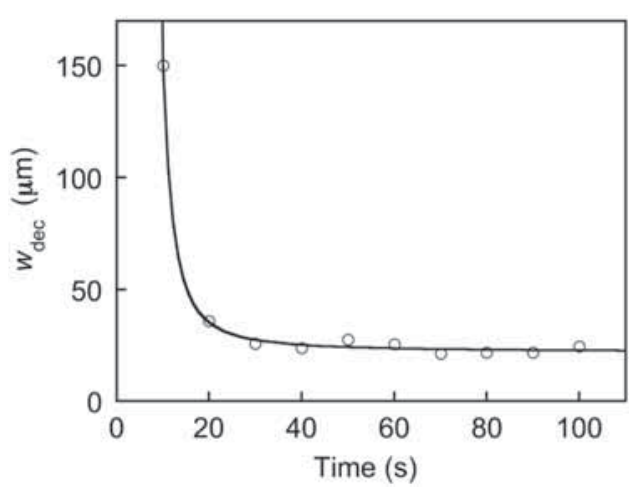

Figure 13. Relationship between rotation time and width of the region where 1-decanol remained. The rotation speed was $600 \mathrm{rpm}$.
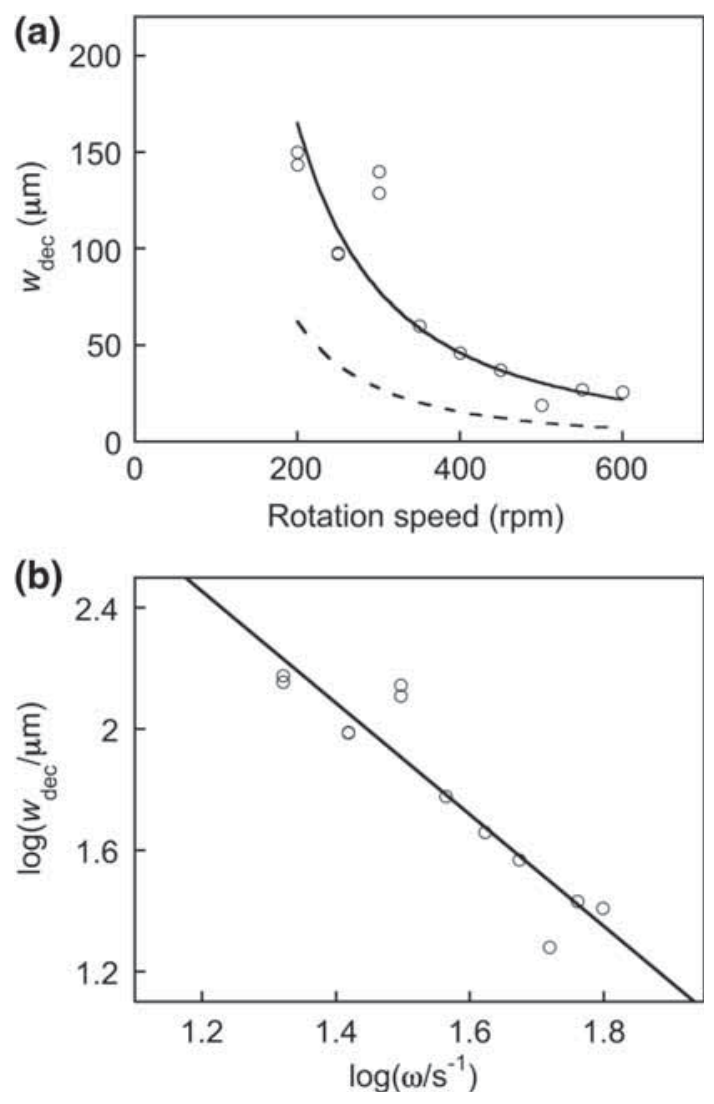

Figure 14. (a) Relationship between rotation speed and width of the region where 1-decanol remained; the broken line was drawn based on a theoretical model; (b) log-log chart based on the data shown in the upper chart.

We tentatively calculated $w_{1}$ using measured contact angles, although a strict quantitative discussion was inadequate because equation (4) was obtained under some assumptions. Table 1 shows the measured advancing and receding contact angles of 1-decanol on two substrates, i.e., the HMDStreated glass slide and the PDMS sheet from which the PDMS blocks were cut out. The average of the advancing contact
Table 1. Advancing and receding contact angles of 1-decanol.

\begin{tabular}{lcc}
\hline Substrate & $\begin{array}{c}\text { Advancing } \\
\text { contact angle } \\
\text { (deg) }\end{array}$ & $\begin{array}{c}\text { Receding } \\
\text { contact angle } \\
\text { (deg) }\end{array}$ \\
\hline HMDS-treated glass slide & 22 & 11 \\
PDMS sheet & 46 & 26 \\
\hline
\end{tabular}

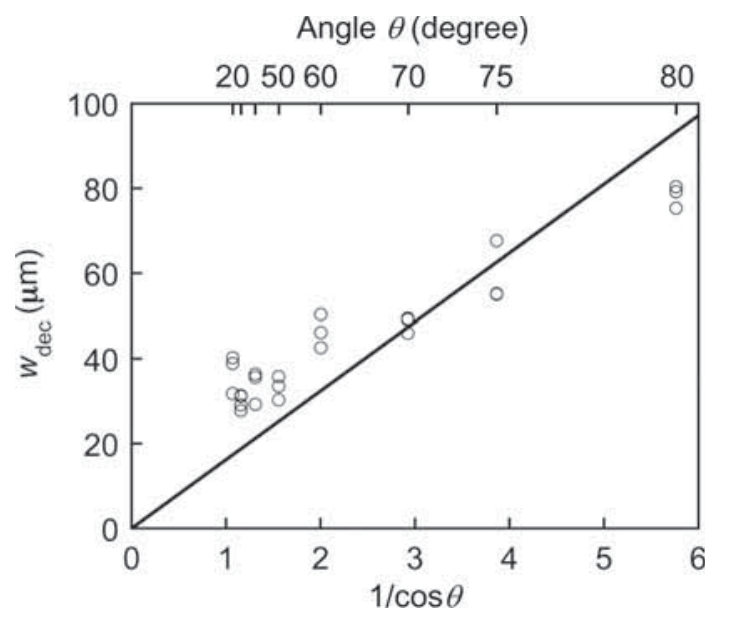

Figure 15. Relationship between the width $w_{\text {dec }}$ of the region where 1-decanol remained and the angle $\theta$ of the PDMS block to the radial direction. Rotation speed and time were $500 \mathrm{rpm}$ and $50 \mathrm{~s}$, respectively.

angles on the HMDS-treated glass slide and that on the PDMS sheet was $34.0^{\circ}$. The average of the receding contact angles was $18.5^{\circ}$. Other values used in the calculation were as follows: $L_{\mathrm{B}}=15 \mathrm{~mm}, \gamma=28.5 \mathrm{mN} \mathrm{m}^{-1}, r=44 \mathrm{~mm}$ and $\rho=0.83 \mathrm{~g} \mathrm{~cm}^{-3}$. The calculated $w_{1}$ is shown by a broken line in figure $14 \mathrm{a}$, which decreases with the increase in rotation speed. This tendency is consistent with the experimental data. However, the calculated values were smaller than the measured ones.

Based on equation (5), one of the possible reasons for this difference can be ascribed to the value of $\cos \theta_{\mathrm{R}}-$ $\cos \theta_{\mathrm{A}}$ because other parameters $\left(\gamma, L_{\mathrm{B}}, \rho, r\right.$ and $\left.\omega\right)$ can be accurately determined. In general, this value can be significantly affected by surface roughness [20]. Although we measured the contact angles on the smoother surface of a PDMS sheet, the block was cutoff from the sheet using a knife. Hence, the side surface of the block was somewhat rough. Therefore, the value of $\cos \theta_{\mathrm{R}}-\cos \theta_{\mathrm{A}}$ in equation (5) might have been smaller than the actual value, and thus, $w_{1}$ was also estimated to be smaller. Another possible reason for the deviation between the theoretical model and the experiments is the previously mentioned assumption that $w_{1}$ is equal to $w_{2}$ (figure 12a). Because the contact angle measured on the PDMS sheet was larger than that measured on the HMDS-treated glass slide (table 1), $w_{1}$ 
Table 2. Static contact angles.

\begin{tabular}{lccc}
\hline No. & Liquid & Substrate & $\begin{array}{c}\text { Contact angle } \\
(\mathrm{deg})\end{array}$ \\
\hline 1 & THF with DBA $(\sim 2 \mathrm{wt} \%)$ & HMDS-treated glass slide & $<10$ \\
2 & THF with DBA $(\sim 2 \mathrm{wt} \%)$ & PDMS sheet & 64 \\
3 & THF & HMDS-treated glass slide & $<10$ \\
4 & THF & PDMS sheet & 56 \\
5 & THF-methanol $(50-50 \mathrm{wt} \%)$ & HMDS-treated glass slide & $<10$ \\
6 & THF-methanol $(50-50 \mathrm{wt} \%)$ & PDMS sheet & 46 \\
\hline
\end{tabular}

should be larger than $w_{2}$. However, we assumed them to be equal. For this reason, the theoretically calculated value $\left(w_{1}\right)$ was smaller than the experimentally measured one $\left(w_{\mathrm{dec}}\right)$.

Based on equation (5), it was predicted that the width $w_{1}$ (or $\left.w_{\mathrm{dec}}\right)$ would be proportional to $1 / \cos \theta$. Therefore, we measured $w_{\text {dec }}$ using the PDMS blocks placed on a substrate with different angles to the radial direction. As shown in figure 15, $w_{\mathrm{dec}}$ was roughly proportional to $1 / \cos \theta$.

As shown in figure 10a, a solution of DBA was used to obtain crystalline needles, instead of 1-decanol. The increase in rotation speed also reduced $L_{\mathrm{DBA}}$. However, the dependence of $L_{\mathrm{DBA}}$ on the rotation speed was not proportional to $\omega^{-2}$. Rather, it was proportional to $\omega^{-0.52}$. The solvent for the DBA solution was a mixture of THF and methanol, whose boiling points ( 66 and $64.7^{\circ} \mathrm{C}$, respectively) are much lower than that of 1-decanol. It was plausible that the removal of excess solvent by the centrifugal force and the evaporation of the solvent proceeded simultaneously. Therefore, the dependence of $L_{\mathrm{DBA}}$ on rotation speed might be different from that of 1-decanol.

Based on the measured receding contact angles of 1-decanol (table 1), the contact angle of the DBA solution droplet on the HMDS-treated glass slide was expected to be smaller as compared with that of the solution droplet on a PDMS sheet. In fact, as shown in table 2, the measured contact angles of the THF solution of DBA (Numbers 1 and 2) or those of pure THF (Numbers 3 and 4 ) are consistent with our expectation. Similarly, in case of a mixture of THF-methanol (50-50 wt\%), the contact angle measured on the HMDS-treated glass slide was smaller than that on the PDMS sheet (Numbers 5 and 6). Because a smaller contact angle leads to a thinner liquid film on a solid surface [19], it was also anticipated that the crystalline DBA needles would preferably precipitate on the glass slide surface rather than on the side of the PDMS block. We examined the block using a light microscope; no crystalline needles were observed on the side of the block, as expected. Thus, the block should be composed of materials with contact angles larger than that measured on the glass slide for preferential deposition of crystalline needles onto the glass slide.

\section{Conclusion}

Using a spin coater and a PDMS block placed on a glass slide, aligned crystalline needles were obtained from solution of an organic compound (e.g., THF solution of DBA). This method required a much smaller amount of solution than that needed in dip coating. Experiments in which a neat liquid organic material, 1-decanol, was used instead of a solution revealed that the liquid was confined in a narrow region on the foot of the PDMS block. This also explained that the amount of the confined liquid was determined by the balance between centrifugal force and capillary force. Therefore, rotation speed could control the amount of the confined solution, and the length of the crystalline needles precipitated from the solution as well. In addition, the thickness of crystalline needles could be controlled by the composition of the solvent. This cost-effective and facile method seems to have potential for use in the fabrication of various electronic devices, such as an array of organic field-induced transistors. In addition, because centrifugal force is expected to increase crystallinity and molecular orientation of polymers [21], this method can potentially be applied for preparing crystalline polymers as well.

\section{References}

[1] Su B, Wu Y and Jiang L 2012 Chem. Soc. Rev. 417832

[2] Tang Q, Jiang L, Tong Y, Li H, Liu Y, Wang Z et al 2008 Adv. Mater. 202947

[3] Zhang X, Lu M, Zhang Y, Chen L and Wang Z L 2009 Adv. Mater. 212767

[4] Pu D, Zhou W, Li Y, Chen J, Chen J, Zhang H et al 2015 RSC Adv. 5100725

[5] Jamal M, Xu J and Razeeb K M 2010 Biosens. Bioelectron. 26 1420

[6] Tang Q, Li H, He M, Hu W, Liu C, Chen K et al 2006 Adv. Mater. 1865

[7] Huang J, Fan R, Connor S and Yang P 2007 Angew. Chem. Int. Ed. 462414

[8] Nam S, Jang J, Anthony J E, Park J, Park C E and Kim K 2013 ACS Appl. Mater. Interfaces 52146

[9] Liu S, Wang W M, Mannsfeld S C B, Locklin J, Erk P, Gomez M et al 2007 Langmuir 237428 
[10] Hong S W, Byun M and Lin Z 2009 Angew. Chem. Int. Ed. 48 512

[11] Messer B, Song J H and Yang P 2000 J Am. Chem. Soc. 122 10232

[12] Tong Y, Tang Q, Lemke H T, Moth-Poulsen K, Westerlund F, Hammershoj P et al 2010 Langmuir 261130

[13] Li H, Tee B C, Cha J J, Cui Y, Chung J W, Lee S Y et al 2012 J. Am. Chem. Soc. 1342760

[14] Deegan R D, Bakajin O, Dupont T F, Huber G, Nagel S R and Witten T A 1997 Nature 389827

[15] $\mathrm{Hu} \mathrm{H}$ and Larson R G 2006 J. Phys. Chem. B 110 7090
[16] Hu H and Larson R G 2002 J. Phys. Chem. B 1061334

[17] Watanabe S, Fujita T, Ribierre J, Takaishi K, Muto T, Adachi C et al 2016 ACS Appl. Mater. Interfaces 817574

[18] Furmidge C G L 1962 J. Colloid Sci. 17309

[19] de Gennes P, Brochard-Wyart F and Quere D 2004 Capillarity and wetting phenomena (New York: Springer-Verlag) Chapter 2, ISBN0-387-00592-7

[20] de Gennes P, Brochard-Wyart F and Quere D 2004 Capillarity and wetting phenomena (New York: Springer-Verlag) Chapter 9, ISBN0-387-00592-7

[21] Shanmuganathan K, Fang Y, Chou D Y, Sparks S, Hibbert J and Ellison C J 2012 ACS Macro Lett. 1960 